\title{
Prospective Usage of the Delta Functions as Possible Software in Piezoelectric Sensors to Measure Instantaneous Signals
}

\author{
Huber Nieto-Chaupis \\ Universidad de Ciencias y Humanidades \\ Center of Research eHealth \\ Av. Universitaria 5175, Los Olivos, Lima39 Perú \\ huber.nieto@gmail.com
}

\author{
Miguel Flores-Vásquez \\ Universidad de Ciencias y Humanidades \\ The Manage Office \\ Av. Universitaria 5175, Los Olivos, Lima39 Perú
}

\begin{abstract}
We review the importance of using a Delta function which can be used as a tool to model instantaneous response signals fact that can be useful to be implemented in software hardware interfaces in the analysis of cracks and breaks in metallic pipes by using piezoelectric sensors. Commonly in an output signal derived from a Dirac-Delta convolution the shape appears to follow the one of the input. When the argument of the Dirac-Delta acquires a polynomial form then the resulting output function contains various peaks in different intensities in according to the polynomial structure of the argument. Thus, a possible application of this formalism would that of the design of algorithms for identifying and measuring the cracks in metallic pipes by using piezoelectric sensors. We found that the usage of the Delta function would serve for simulating the reconstructed spectrum in display with a data acquisition much more fast that the conventional ones. This methodology might be exploited in the analysis of super fast signals which need to be measure with acceptable resolutions.
\end{abstract}

\section{INTRODUCTION}

It is well-known that the Delta functions are introduced as examples of input functions representing very instantaneous impulses but with a well-defined output signal which can be well measured in the frequency domain for example. Some interesting properties of the Delta function might be of importance in the extension of mathematical methodologies aimed to extract information of systems whose intensity of inputs signals is limited in time[1]. Normally, Delta functions contain linear argument and their inclusion in convolution integrals makes the result trivial as seen in abundant examples in literature. Indeed the Delta functions are useful in many applications of the Physics and Mathematics because it provides simplification in calculating operations of the type I/O. On the other hand, it serves as a medium for calculating integrals with a dependence multi-variable [2]. In this note, the point that the usage of a Delta functions containing a polynomial function in their arguments is briefly boarded. Our view might be of importance in those cases where a modeling of distorted output signal created by instantaneous input functions is needed [3][4][5]. Of great importance for this paper is the case when the Delta functions are convoluted together with a orthogonal polynomials. By means of simple calculations it is shown that the quadratic argument makes the output morphology much more complex as viewed in the apparition of secondary harmonics and thereby reducing the amplitude below one half of its value when no polynomial expressions are used inside the Delta functions. Apart of that, output curves also show that a shift of the first harmonic occurs as well. This paper is structured as follows: in second section we review the mechanisms of integration and convolution[6][7][8][9] with the Delta functions. In third section, it is examined the inclusion of Delta functions with polynomial argument together with their kernels associated by which the Bessel functions are tested. Here is presented the curves obtained with the package Mathematica Wolfram [10] comparing the cases with and without the polynomial argument. In fourth section we stress the applicability of this formalism for studying the dynamics of cracking in metallics pipes and its prospects in performing simulations with algorithms associated. Finally in last section, the conclusion regarding the results of this paper is drawn.

\section{LineaR AND Nonlinear InTEGRALS WITH DELTA FUNCTIONS}

The well-known Dirac-Delta functions are established on the basis that transfer the dependence variable through the integration for example: the well-known linear case is given by $\int_{\infty}^{\infty} \delta(t-\tau) x(\tau)=y(t)$. For instance, this scheme can be applied in those system identification schemes which are defined at least with diagonal terms in some truncated series (Wiener or Volterra). For instance,

$y(t)=\int h(\tau) x(t-\tau) d \tau+\iint h(\tau) h(\beta) x(t-\tau) x(t-\beta)$.

When Delta functions replace the input functions the resulting integration turns out to be a triviality. Clearly the output depends on the morphology of the kernels $h$,

$$
\begin{gathered}
y(t)=\int h(\tau) \delta(t-\tau) d \tau+\iint h(\tau) h(\beta) \delta(t-\tau) \delta(t-\beta) d \tau d \beta \\
y(t)=h(t)+h^{2}(t)
\end{gathered}
$$


One can see that the usage of kernels like the so-called orthogonal polynomial would give a defined shape to the output $y(t)$. So that depending on the $N$-order of approximation then $y(t)=\sum_{k}^{N} h^{k}$.

\section{A. The Nonlinear Case}

The nonlinear case is defined as the convolution integral contains a Delta function [11][12] with a polynomial argument fact that makes us to introduce free parameters by which to some extent it might be understood as the system identification parameters[13][14], so that it reads

$$
y(t)=\int d \tau h\left(\tau_{1}\right) \delta\left(G_{1}\left(\tau_{1}, t\right)\right)
$$

where now the Delta function argument express the fact that it depends on a generalized $G_{1}\left(\tau_{1}, t\right)$ function that encloses a high degree polynomial. For instance a quartic degree polynomial can be written as

$$
G_{1}\left(\tau_{1}, t\right)=\left(\tau_{1}-\alpha_{1} t-\alpha_{2} t^{2}\right)\left(\tau_{1}-\beta_{1} t-\beta_{2} t^{2}\right) .
$$

\section{RAPid Fall Oscillating of the Delta Convolutions}

The well-known and so-called spike function of the Delta functions can be written in their most generalized form as

$$
\delta[f(\tau)]=\delta\left[\prod_{i=1}^{N}\left(\tau-a_{i}\right)\right]=\sum_{i=1}^{N} \frac{\delta\left(\tau-a_{i}\right)}{\left|\frac{d f(\tau)}{d \tau}\right|_{\tau=a_{i}}}
$$

where $f(\tau)=\left(\tau-a_{1}\right)\left(\tau-a_{2}\right)\left(\tau-a_{3}\right) \ldots\left(\tau-a_{N-1}\right)\left(\tau-a_{N}\right)$ is a continue function whose zeros are denoted by the real numbers $a_{1}, \ldots, a_{N}$. It is clear that $f(\tau)$ is of order " $N "$. The case of interest is when the argument of the Delta function (left side) is written as

$$
\delta[f(\tau-g(t))]=\sum_{\text {zeros }}^{N} \frac{\delta(\tau-s(t))}{\left|\frac{d f(\tau-g(t))}{d \tau}\right|_{\tau=\text { zeros }}}
$$

thereby including a function $s(t)$ which features the "spike" property as expressed in (6). Four remarks:

- Firstly, only is considered the case where the function $f$ depends only on $\tau$ but not polynomials in $\tau$.

- Secondly, the resulting function $s(t)$ depends uniquely on $t$ but it does not contains mixed terms like $\tau t$ (One can see that everything would completely different if one assumes mixed terms, but in praxis it would led us to non physical scenarios without practical applications).

- Thirdly, the first derivate as observed on the denominator of (6) automatically provides a polynomial term in the variable " $t$ ".

- Fourthly, the dependence of a polynomial in $t$ in the denominator as see in (7), it is in advance telling us that any function used in a convolution integral together to Delta functions with polynomial argument, would change drastically in shape and amplitude in according to the free parameters of the Delta function argument.
Our analysis considers as main assumption the fact that the power intensity amplitude undergoes constantly degradation as effect of the presence of multiple obstacles and terrain morphologies. A candidate model ad hoc is that of the I/O integral, expressed by the well-known the Dirac-Delta convolution, $f(r)=\int_{-\infty}^{\infty} f(x) \delta[g(x)] d x$ where $g(x)=\prod_{i=1}^{N}\left(x-a_{i}\right)$, with $a_{i} \in \mathcal{R}$ a $N$-grade polynomial which apparently seems to be difficult, but can easily be solved by using the so-called 'spike' property [5]

$$
\delta[g(x)]=\sum_{i=1}^{N} \frac{\delta\left(x-a_{i}\right)}{\left.\frac{d g(x)}{d x}\right|_{x=a_{i}}} .
$$

For example, let us consider the most simple application $\int f(x) \delta\left(x^{2}-r^{2}\right) d x \approx f(r) / 2 r$ and generalized to $\int f(x) \delta\left(x^{m}-r^{n}\right) d x \approx 1 / r^{n-1}$. It indicates that the resulting denominator is correlated with the argument of the Dirac-Delta functions. It is also noted that the denominator absorbs the rest of variables from the Dirac-Delta function. All these ideas allows us to define in Table I the following forms of arguments in the Dirac-Delta functions in the sense that $g(x) \rightarrow g(x, r)$. These cases were already studied in [5]. The present study

TABLE I

VARIOUS TYPES OF ARGUMENTS OF THE DIRAC-DELTA FUNCTION.

\begin{tabular}{|c|c|c|}
\hline Name & $\prod_{k=1}^{Q} g(x, r)$ & Number of Parameters \\
\hline \hline uniform & $x-r-\lambda_{k}$ & $\mathrm{Q}$ \\
\hline composed & $b_{k} x-a_{k} r-\lambda_{k}$ & 3Q \\
\hline mixed & $\mu_{k} r x-a_{k} r-b_{k} x-\lambda_{k}$ & 4Q \\
\hline functional & $\mu_{k} r x-h\left(r, a_{k}\right)-b_{k} x-\lambda_{k}$ & 4Q+Q' (Q' $>1)$ \\
\hline
\end{tabular}

has paid attention to the 'mixed' case where is assumed that the system contains $4 \mathrm{Q}$ free parameters. An interesting application of this scenario is that of the convolution of any observable together with the Dirac-Delta function. Under this view, one expects change of morphology of the resulting output functions. For instance, given a continuous function $f(x)$ then $\forall x \subset \mathcal{R}$ and $f \subset \mathcal{R}$ and the mixed case of table I then the Dirac-Delta convolution written as

$$
\mathcal{F}(r)=\int_{-\infty}^{\infty} \delta[g(r, x)] f(x) d x
$$

where $g(x, r)=\prod_{k=1}^{M}\left(\mu_{k} r x-a_{k} r-b_{k} x-\lambda_{k}\right)$ with $r \in \mathcal{R}$ is a continuous variable, and $\mu_{k}, \lambda_{k}, a$, and $b \in \mathcal{R}$ has as result the following closed-form expression

$$
\begin{aligned}
& \int_{-\infty}^{\infty} \sum_{q=1}^{N}\left[\frac{\delta\left(u-\beta_{q}\right)}{\prod_{m}^{N} \sum_{n}^{N}\left[\frac{u-\beta_{m}}{u-\beta_{n}}\right]}\right] f\left(\frac{u}{\gamma}\right) \frac{d u}{\gamma}, \\
= & \sum_{q=1}^{N} \int_{-\infty}^{\infty} \frac{1}{\gamma}\left[\frac{\delta\left(u-\beta_{q}\right)}{\prod_{m}^{N} \sum_{n}^{N}\left[\frac{u-\beta_{m}}{u-\beta_{n}}\right]}\right] f\left(\frac{u}{\gamma}\right) d u,
\end{aligned}
$$

It is easy to recognize that the integration is just reduced to the most simple application of the Dirac-Delta convolution and its 


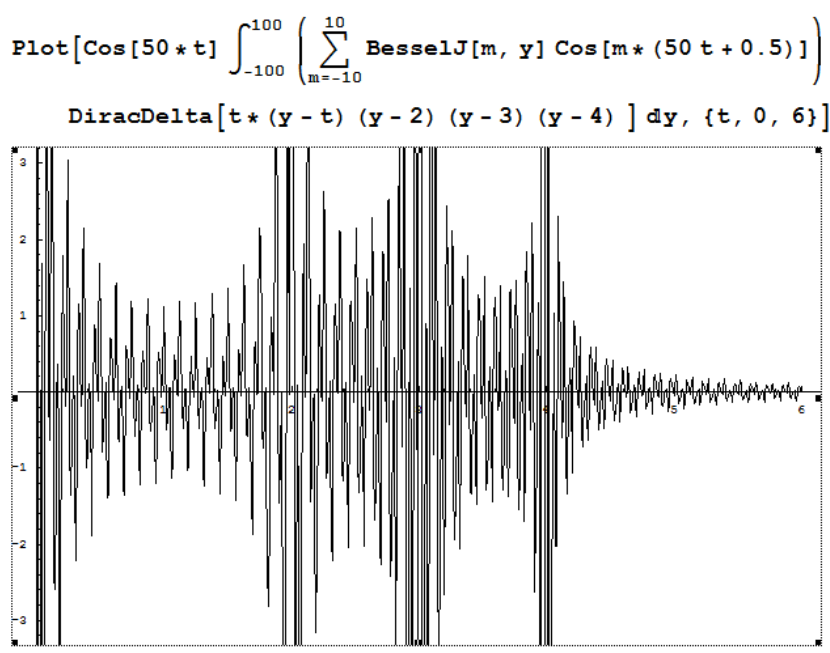

Fig. 1. Illustration of Eq.13 indicating the steps done with the package Wolfram Mathematica [10]. Here the argument has been of order of fifth degree in $t$.

evaluation makes it handleable, thus the after the integration one can verify that

$$
\begin{gathered}
=\sum_{q=1}^{N}\left[\frac{1}{\prod_{m}^{N} \sum_{n}^{N}\left[\frac{\beta_{q}-\beta_{m}}{\beta_{q}-\beta_{n}}\right]} \frac{1}{\gamma} f\left(\frac{\beta_{q}}{\gamma}\right)\right] \\
=\sum_{q=1}^{N}\left[\frac{1}{\prod_{m}^{N} \sum_{n}^{N}\left[\frac{\frac{a_{q} r+\lambda_{q}}{\mu_{q} r-b_{q}}-\frac{a_{m} r+\lambda_{m}}{\mu_{m} r-b}}{\frac{a_{q} r+\lambda_{q}}{\mu_{q} r-b_{q}}-\frac{a_{n} r+\lambda_{n}}{\mu_{n} r-b_{n}}}\right]} \frac{1}{\gamma} f\left(\frac{a_{q} r+\lambda_{q}}{\mu_{q} r-b_{q}}\right)\right]
\end{gathered}
$$

that denotes a compact manner of the resulting usage of the Delta functions.

\section{A. Integration Results}

The plotting of spectra with and without polynomial argument is given with the help of the software Mathematica[10], as shown in Fig 1. It is quite interesting to note the presence of additional harmonics. The syntax of the commands above the plot follows the one of Wolfram. Here is indicated the convolution of a cos function together with a integer-order Bessel function resulting in a fully function. Here is considered the sum of all integer inside the range -10 and 10. The argument is proportional to $y^{4}$. In Fig. 2 is shown the cases where on order of the Bessel function is taken inside of a Volterra-like convolutions for second and third order. Top panel the case of a quadratic Bessel function whereas bottom indicates the cubic as seen as the product of up to three Bessel functions. Although it does not changes dramatically the spectra one sees that the principal as well as the secondary harmonics are shifted. Besides, the amplitude is degraded by a substantial fraction by making negligible the value of the "wave" in large values of $t$ (independent variable). In other words, the inclusion of quadratic terms of $t$ has the direct effect

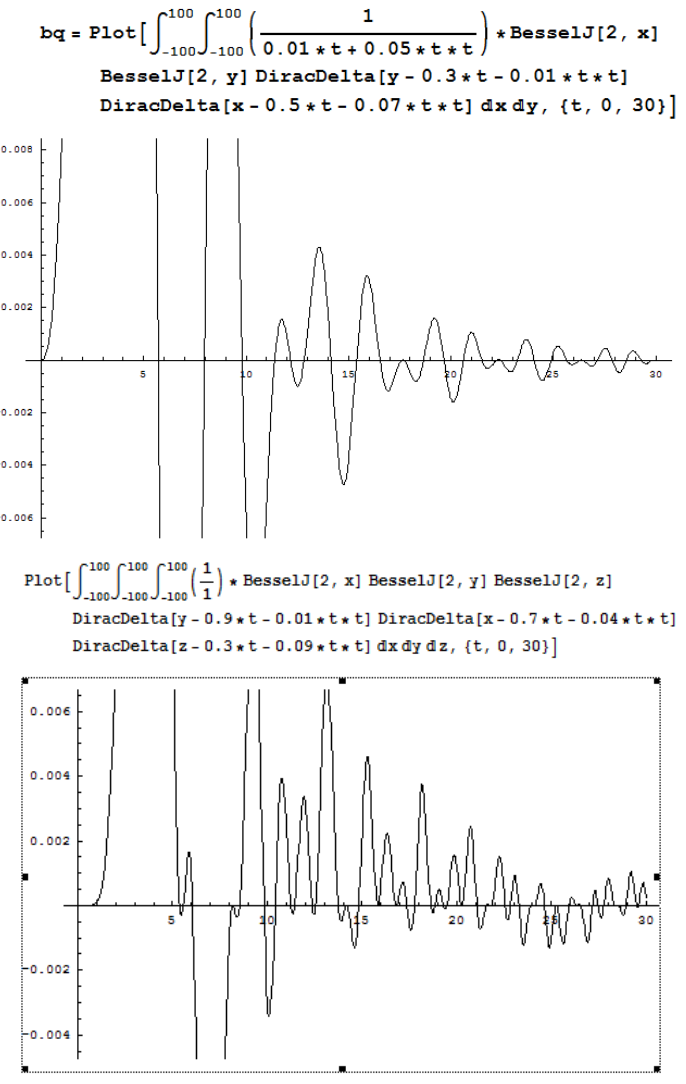

Fig. 2. Top left (right) panel: the case of the quadratic contributions in Bessel functions and Delta also. Bottom: contribution of up to third degree, cubic terms.

in decreasing the wave over the horizon until its disappearance. The free parameters inside the argument is also another factor to modify the shape of resulting curves. In Fig 3 up to three are displayed. While top and are obtained with Eq. 13, bottom plot used Eq. 9 as the uniform case in according to Table I.

\section{APPLICABILITY FOR IDENTIFYING AND ASSESSING CRACKS IN PIPES}

A potential usage of this formalism would be that of monitoring the damage caused by aging and first stages of fracture in metallic pipes. Interestingly, a possible application of those schemes would be in the case of nonlinear response containing a high degree of distortions as consequence of power amplifiers. Another potential usage of this formalism would be that of monitoring the damage caused by aging and first stages of fracture in metallic pipes. In fact, in figure 4 is represented the manner how Delta convolution integrals within the I/O formalism is employed. Whereas the apparition of cracks plays a negative role for deteriorating pipes systems aimed to transport gas and oil, it is important a preventive detection with advanced tools by allowing the location and identification in time. In figure 4 the applicability of this formalism is seen as a sequence. At the top is denoted the case of a solid with the apparition of two cracks. To note the cartoon displaying two cracks of different intensity. Both 


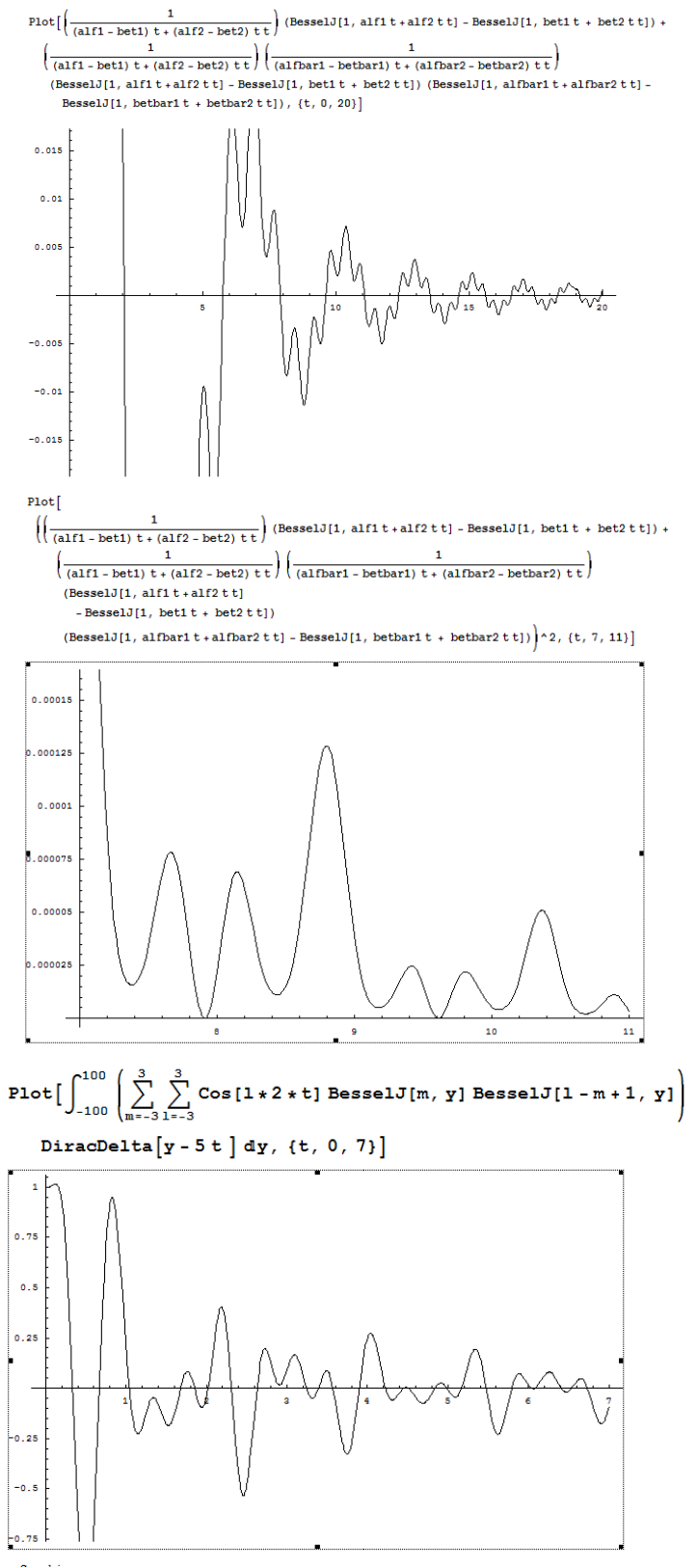

Fig. 3. Top and Middle: different plots when the using directly Eq.13. Bottom uses the "uniform" case in according to Table I: The resulting output when the Delta function is used in the programming with Mathematica [10].

are scanned by the emission and detection of a pulse. One should expect the phenomenon as shown at the middle which anticipates the register of just a spectrum composed by two peaks in coherence with the cracks. The lowest cartoon is the reconstructed spectrum after the processing by using the algorithm containing the Delta convolution. The convolution operation $y(t)=\int \delta(t-G(t, \tau)) h(\tau) d \tau$ is then applied by reducing the amplitude size with the subsequent apparition of second harmonics. The whole spectrum is shifted as well. To note that $\tau_{2} ; \tau_{1}$ which is necessary for the processing acquisition data from the piezoelectric detector. It clearly

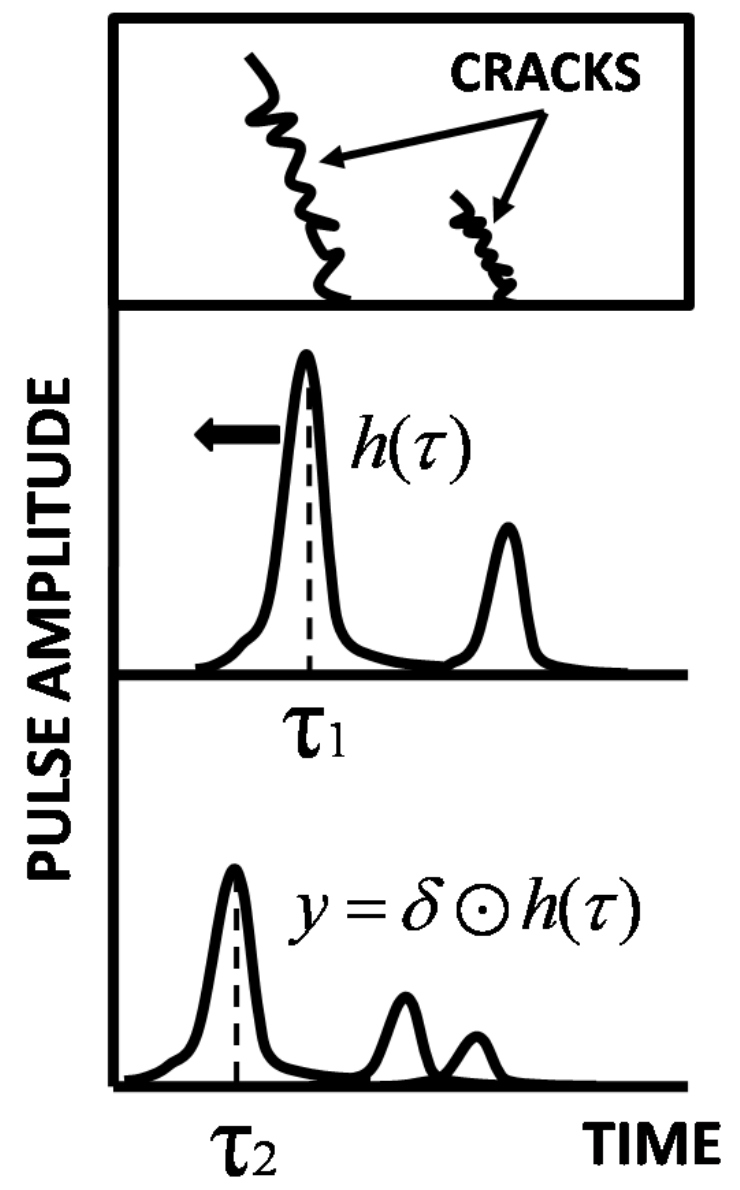

Fig. 4. Interpretation and applicability of the Delta function with polynomial algorithms in convolution integrals is sketched. The cracks are analyzed and detected by a piezoelectric device as shown in upper. In the middle the output signals are taped through a piezoelectric detector given the two peaks by showing different amplitudes by effect of the two cracks. To note that the spectrum denoted by $h(\tau)$ is peaked around $\tau_{1}$ before hardware processing. Below is displayed the final spectrum showing that the processing taking into account the Delta convolution methodology has shifted to left the first peak and reducing substantially the amplitude size. So that one can observe the advantage in processing much more fast with a dedicate resolution to some extent.

constitutes a notable advantage in comparison with known ordinary techniques.

\section{A. Parameters Identification in Pipes}

It is possible to perform identification of the pipe damage by effect of crack evolution [15] by using the Delta convolution model. The parameters to be identified would that of the Delta argument. For this exercise two cases are tested:

$$
G_{A}\left(\tau_{1}, t\right)=\left(\tau_{1}-\alpha_{1} t\right)\left(\tau_{1}-\beta_{1} t\right),
$$

and a second nonlinear approximation, in virtue to Eq.5,

$$
G_{B}\left(\tau_{1}, t\right)=\left(\tau_{1}-\alpha_{1} t-\alpha_{2} t^{2}\right)\left(\tau_{1}-\beta_{1} t-\beta_{2} t^{2}\right) .
$$




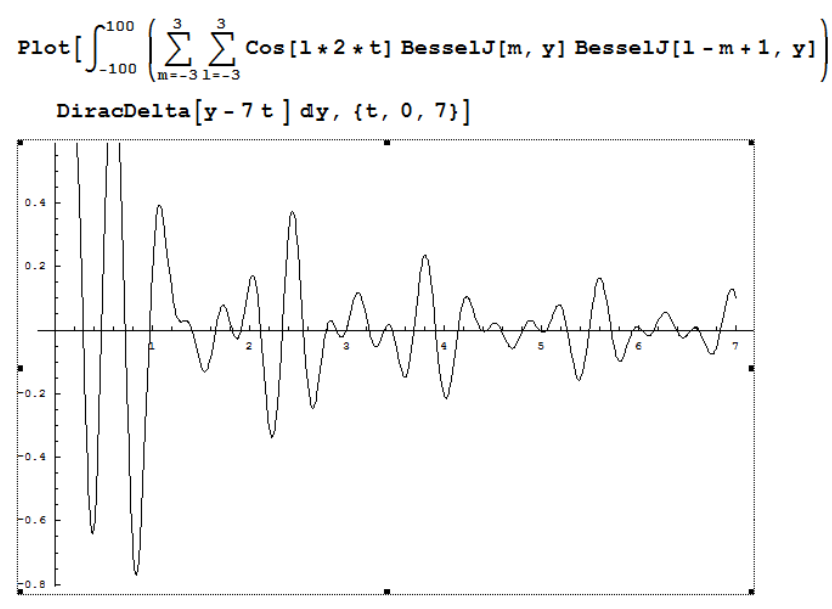

Fig. 5. Fully distorted spectrum from an input function given by a quadratic Bessel function and a cos.

In figure 5 we have extracted the values $a=1$ and $b=-7$ the composed case in according to Table I. All values are consistent over the integration range between -100 to 100 . We defined crack in between 0.5 and 1 where is theoretically assumed resulting in a reconstructed spectrum of 0.15 and the peak being shifted from 5.4 to 6. Minor harmonics are appear but they are displaced to right. In praxis one can normalize the resulting spectrum on mean values on software.

\section{B. Interpretation of the Eq. 9 as Physical Output Due to Instantaneous Impulse}

A point that should be stressed, is the possible presence of poles or discontinuities because the zeros of the denominator of Eq.(13). The present methodology opts the most optimal configuration of free parameters whose resulting function $\mathcal{F}(r)$ must a real number. In Fig. 5 and Fig. 6 two plots obtained from direct evaluation of Eq.(9), are displayed. One can see that the usage of a very few parameters makes impact on the morphology of curve. For instance, in Fig. 6 top a dip is seen in 6 . Actually the values of $a$ and $b$ parameters make the creation of a contiguous dip. It makes us to suppose that the $a$ and $b$ parameters are entirely responsible to create successive dips as is demonstrated in Fig. 6. For example, the apparition of multiple dips can be associated to physical elements whose end is to decrease the curve amplitude as simple effect of scattering. In others words, the 'zeros' of Dirac-Delta function can be associated to space points where the intensity of a fields falls to distance or is near to be null. Mathematically speaking, when parameters acquire higher values, the nonlinearity is observed in the successive generation of oscillations. This fact is quite interesting in order to interpret it inside a physics scenario. In bottom panel of Fig. 6 is seen a kind of aperture or crack just to be recognized by a piezoelectric sensor (see for instance a methodology as given in [15]). The $g(\tau)$ is cubic. All this phenomenology is best seen in Fig. 7 where cracks and breaks have been interpreted when the function $f$ in Eq.(9) depends of up to 2 independent variables. In top and middle

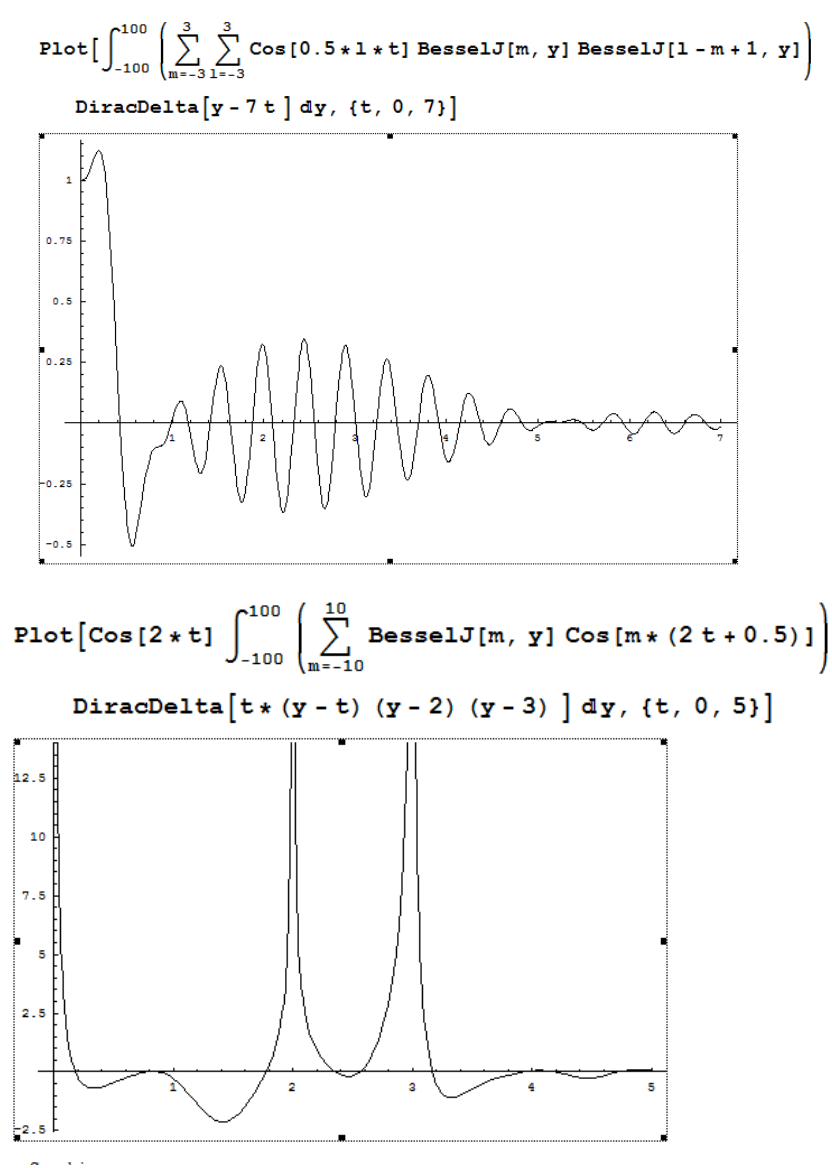

Fig. 6. Top: direct integration of Eq, 9 with mathematica. Bottom: exact identification of a break as seen the apparition of two large pronounced peaks.

plots are seen the cases of possible cracks whereas bottom panel the case of a break. Clearly the difference between crack and break is the value of the length of two successive peaks as seen in bottom Fig. 6. Thus in Fig.8 is seen only the case of break when the cos function depends on $u$ and $t$ while the Bessel function only depends on the variable of integration $y$. The breaks are recognized by the value or distance between two adjacent peaks.

\section{CONCLUSION}

In this note we have testing the case when Delta functions contain in their argument a kind of polynomial, fact which is observed when are inserted in I/O relations in conjunction to self interactions. The results can be an option to model complex signals with a higher grade of distortion by effect of unknown mechanisms or interference. The formalism is tested in the identification of cracks in metallic pipes which normally suffer of aging and mechanical deformations for longer use. In future, we shall board the problem of covering all orthogonal families to characterize output functions.

\section{REFERENCES}

[1] Huber Nieto-Chaupis, "Modeling and Monte Carlo simulation of call completion success probabilities under the input-output scheme", Ecuador 


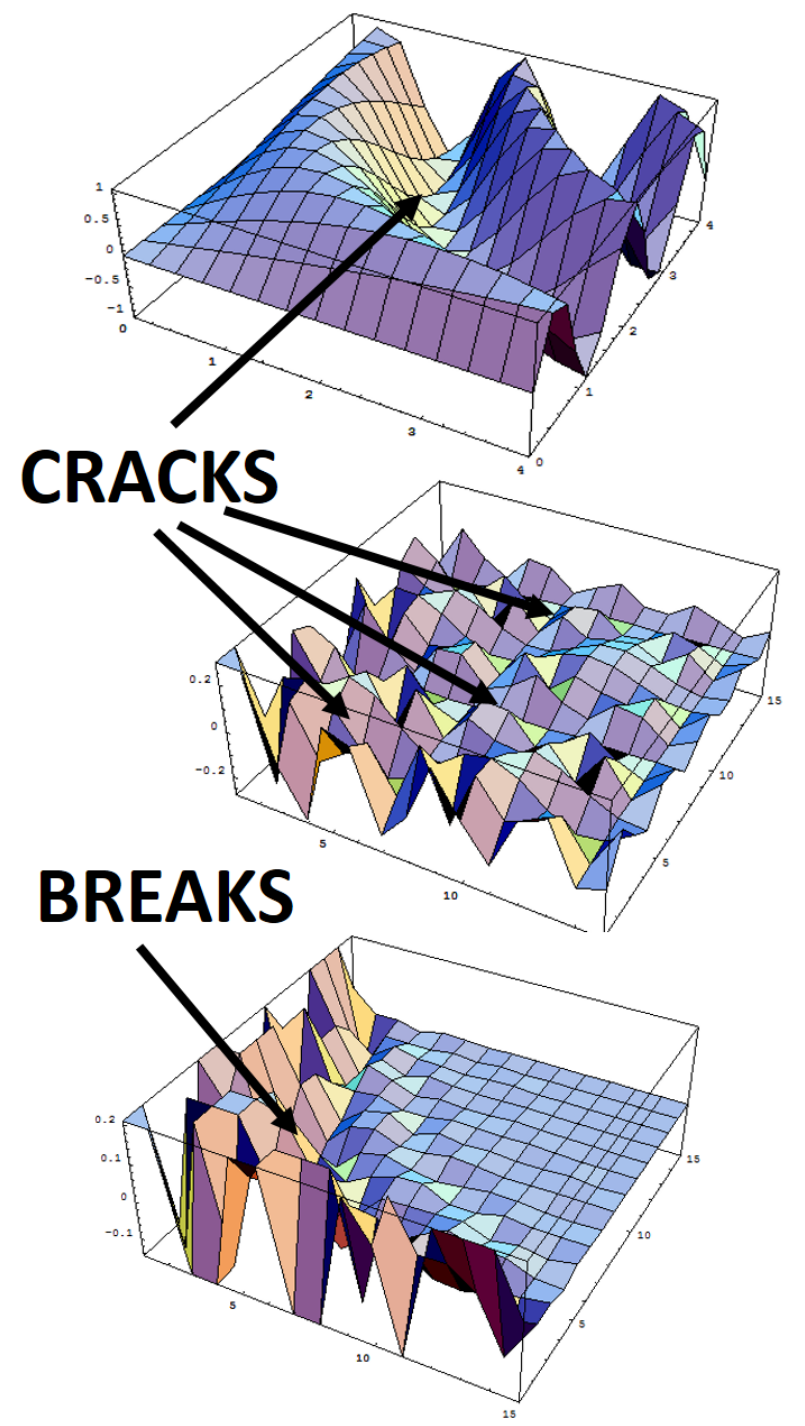

Fig. 7. Examples of cracks and breaks assumed to be sensed by a piezoelectric material. Clearly a break is seen as the substantial difference of amplitudes between two adjacent peaks.

Technical Chapters Meeting (ETCM) IEEE, pp. 1-5, 2016. View Article Full Text: PDF (503KB)

[2] Huber Nieto-Chaupis, "Generalization of the classical delay-and-sum technique by using nonlinear dirac-delta functions", Electronics Electrical Engineering and Computing (INTERCON) 2017 IEEE XXIV International Conference on, pp. 1-4, 2017. View Article Full Text: PDF (269KB)

[3] H. Nieto-Chaupis, The Dirac-Delta Functions for Signal Path-Loss Modeling at Mid-Sized Urban Areas, RFID Sao Paulo, Brazil:, September 2014.

[4] Huber Nieto-Chaupis, "Measurement of the probability of call completion with the Dirac-Delta convolution", Automatica (ICA-ACCA) IEEE International Conference on, pp. 1-4, 2016. View Article Full Text: PDF $(1175 \mathrm{~KB})$

[5] Huber Nieto-Chaupis, A closed-form expression for the received power through the usage of the Dirac-Delta functions with nonlinear argument, 2015 International Workshop on Telecommunications (IWT), 4-17 June 2015, Santa Rita do Sapucai, Brazil.

[6] H. Nieto-Chaupis,"On the usage of Dirac-Delta functions with nonlinear argument in high order I/O integrals", Proc. 2014 IEEE Int. Instrumentation and Measurement Conference (I2MTC) Montevideo, Int. Specialized Book Service, pp. 519-524, 2014
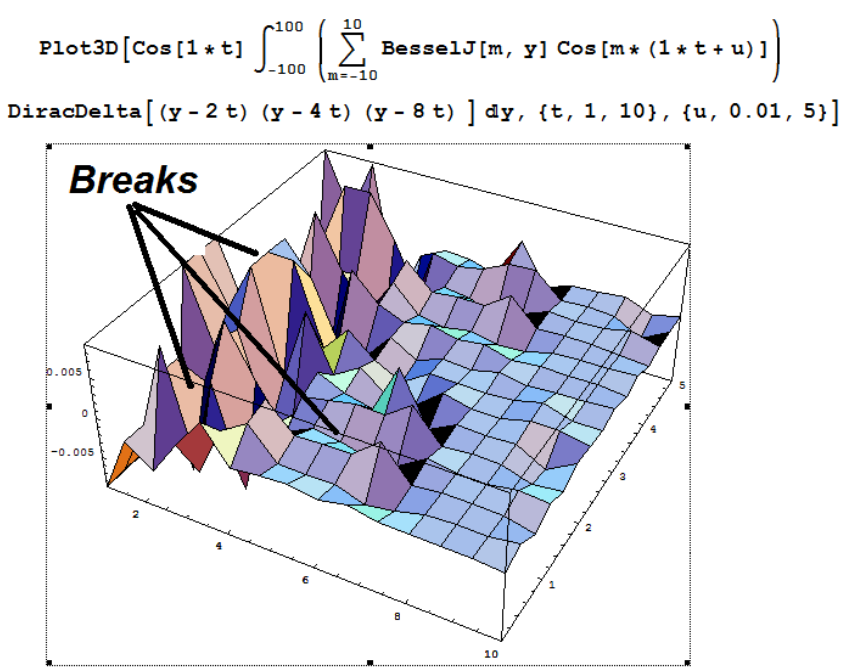

Fig. 8. Example of breaks with a uniform case of the Delta function when the input function is the multiplication of a Bessel function by a cos.

[7] Hoskins R. F., Delta Funtions: Introduction to Generalized Functions 2nd Edition, Int. Specialized Book Service.

[8] Jon Mathews and R. L. Walker, Mathematical Methods of Physics, 2nd ed., Addison-Wesley Publishing Company, Inc., pp.6873.

[9] Won Mee Jang, Quantifying Performance in Fading Channels Using the Sampling Property of a Delta Function, Communications Letters IEEE, Vol 15, pp.266-268 (2011).

[10] Mathematica Wolfram Version 4.1: www.wolfram.com.

[11] R. Courant and D. Hilbert, Methods of Mathematics Physics (2 Vols.), Wiley (Interscience), New York, (1966).

[12] Arfken, W., Mathematical Methods for Physicists, 7th Edition Academic Press.

[13] J. Goodman, M. Herman, B. Bond and B. Miller "A Log-Frequency Approach to the Identification of the Wiener-Hammerstein Model", IEEE Signal processing Letters Vol. 16, pp 889-892, 2009.

[14] L. Ding et.al "A robust digital baseband predistorter constructed using memory polynomials" IEEE Trans. Commun., pp. 159-165, 2004.

[15] Y. Han ; A.K. Misra ; D. Mateescu, A method for crack detection in structures using piezoelectric sensors and actuators, 2005 International Conference on MEMS,NANO and Smart Systems, 24-27 July 2005, Banff, Alta., Canada, Canada. 